\section{Isolation of (-)-Epigallocatechin from Green Tea Extract by Means of Immobilized Tannase}

\section{Min Li, Brendan T. Kelly, Ann E. Hagerman}

Department of Chemistry \& Biochemistry, Miami University, Oxford, $\mathrm{OH}$, United States

\begin{abstract}
$\nabla$

(-)-Epigallocatechin is a chemoprotective polyphenolic compound found in green tea. Its low abundance in tea increases the cost and difficulty of obtaining sufficient (-)-epigallocatechin to conduct research on (-)-epigallocatechin-mediated health benefits. We developed a convenient and economical method for preparing (-)-epigallocatechin from a commercial green tea extract in a sufficient quantity to carry out mechanistic studies of the pure compound. Crude epigallocatechin gallate was isolated from the tea extract by silica gel column chromatography, and was then hydrolyzed with immobilized tannase. The (-)-epigallocatechin found in the hydrolyzed products was extracted into ethyl acetate and purified by Sephadex LH-20 to produce (-)-epigallocatechin. Very pure (-)-epigallocatechin (93.7\%) was obtained and its identity was confirmed by ESI-MS and HPLC. The overall yield was $10 \%$ by mass of the initial green tea extract obtained as pure (-)-epigallocatechin. No specialized equipment was required, and the solvents and chromatographic supports used were inexpensive and readily available.
\end{abstract}

Key words

Camellia sinensis $\cdot$ Theaceae $\cdot$ catechin $\cdot$ flavonoid $\cdot$ polyphenol . antioxidant $\cdot$ tea

\section{Abbreviations \\ $\nabla$ \\ EGC: (-)-epigallocatechin \\ EGCg: (-)-epigallocatechin gallate}

EGC is a polyphenolic compound commonly found in tea, Camellia sinensis L. (Theaceae). Polyphenols including flavonoids may contribute to the health-promoting benefits of plant-based foods and beverages such as tea via bioactivities such as antioxidant activity, protein precipitation, and metal chelation [1-3]. However, identifying the compounds responsible for specific bioactivities has been difficult, in part due to the expense of some purified flavonoids, particularly those like EGC that are minor constituents of complex mixtures in natural sources [4].

Although methods have been developed for isolating EGC directly from tea, two factors limit their application [5]. One is the very low content of EGC in green tea. The other limitation is an instrumental requirement. Although fractions enriched in specific catechins can be prepared by solvent extraction [6], most methods for the purification of EGC use countercurrent chromatography [4,7-9]. This instrumentation is relatively uncommon, and thus many laboratories cannot obtain pure EGC using existing methods. We developed a method for the preparation of EGC that is convenient and economical, and relies only on conventional extractions and chromatography.
We chose to use a commercial green tea extract as a convenient source of EGCg, which can be hydrolyzed to yield the desired product, EGC ( $\bullet$ Fig. 1). The green tea extract is rich in EGCg ( Fig. 2 a), and $85 \%$ of the original preparation is soluble in ethanol. We used silica gel chromatography to fractionate the ethanol solution, recovering about two-thirds of the initial green tea extract as EGCg ( $\bullet$ Table 1 and Fig. 2 b).

EGCg was hydrolyzed into EGC and gallic acid by immobilized tannase. The reaction was allowed to proceed for $30 \mathrm{~min}$ to obtain the maximal amount of EGC. Although some unhydrolyzed EGCg remained in the product, longer hydrolysis resulted in the loss of EGC to undefined side products. By using the enzyme incorporated into alginate beads, it was easy to recover the polyphenols from the enzyme by ethyl acetate extraction ( $\bullet$ Fig. 2c). The ethyl acetate extraction was important for EGC enrichment, enhancing the purity 4.5-fold compared to samples that were not extracted. During the hydrolysis, some tannase beads appeared to dissolve in the reaction buffer, diminishing the purity of the product. Ethyl acetate extraction helps separate the desired product from the very water-soluble alginates that comprise the tannase beads [10]. In addition, the solubility of gallic acid is much higher in water than in ethyl acetate [11], so extraction in ethyl acetate helped remove the undesirable product gallic acid from the EGC. About half of the EGCg was recovered as EGC after the hydrolysis and ethyl acetate fractionation ( Table 1).

EGC was further purified by Sephadex LH-20 chromatography, using slightly acidified aqueous ethanol as the mobile phase to stabilize EGC [12]. Three peaks eluted from the column, and were identified by their retention times on HPLC and their characteristic UV-visible features ( Fig. 3). About $10 \%$ of the starting crude green tea extract was recovered as EGC ( $\odot$ Table 1 ).

The purity of the isolated EGC was $93.7 \%$ based on HPLC analysis (○ Fig. 2d). The HPLC retention time ( $t_{R} 19.6 \mathrm{~min}$ ) for our purified compound was identical to authentic EGC (Sigma-Aldrich). The UV spectrum of the EGC peak has a high $A_{220}$ to $A_{280}$ ratio that is quite distinct from the UV spectrum of GA or EGCg ( $\bullet$ Fig. 2). The characteristic molecular ion peak $(\mathrm{m} / \mathrm{z} 305)$ is the dominant peak in the mass spectrum ( $\odot$ Fig. 4). A peak at $m / z 611$ was an EGC dimer based on the clear anion signal for EGC $(m / z 305)$ in the MS/MS spectrum. Both the MS spectrum ( Fig. 4) and the MS/ MS spectrum of the parent ion matched that of an authentic standard [13]. No signal was detected for $\operatorname{EGCg}(m / z 458)$ or gallic acid

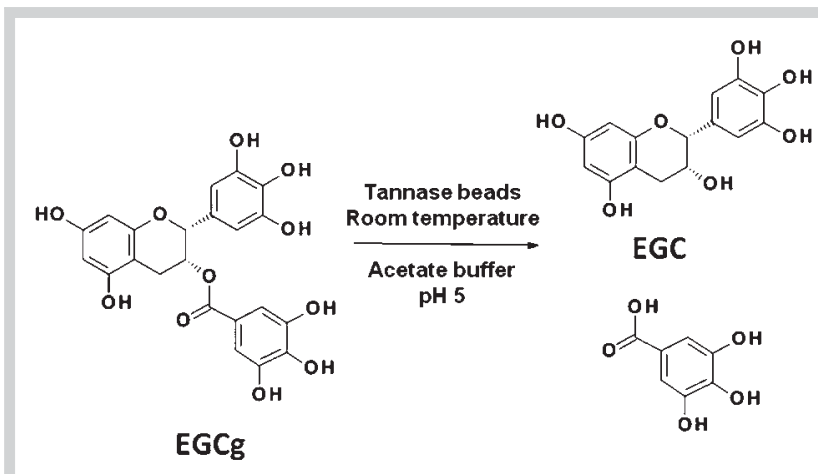

Gallic acid

Fig. 1 Structural formulas of EGCg and its hydrolysis products EGC and gallic acid. 


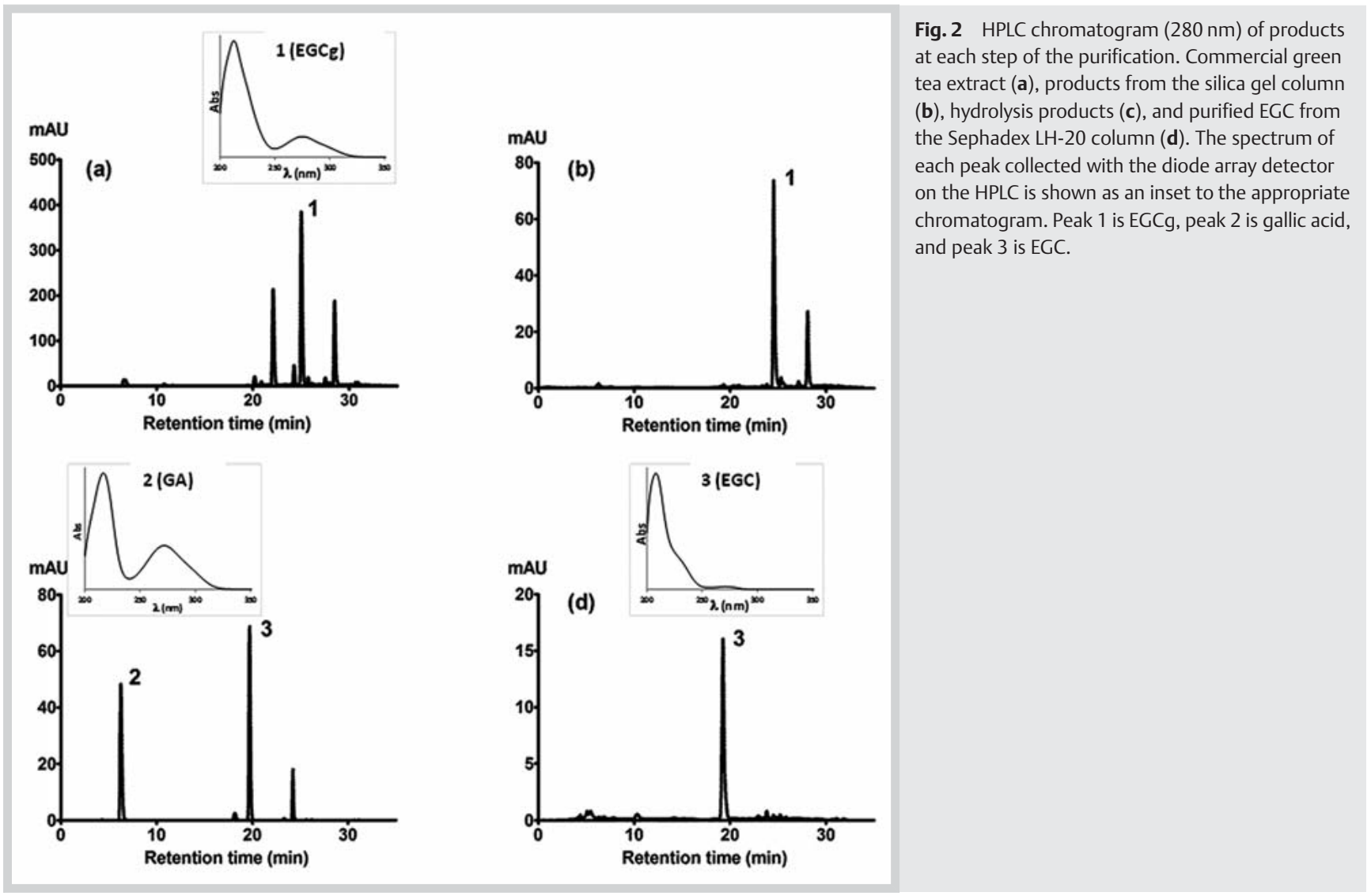

Table 1 Yield and purity of samples from each preparation step.

\begin{tabular}{|c|c|c|c|}
\hline & $\begin{array}{l}\text { EGCg from } \\
\text { silica gel }\end{array}$ & $\begin{array}{l}\text { EGC in hydrolysis } \\
\text { products }\end{array}$ & $\begin{array}{l}\text { EGC from } \\
\text { Sephadex LH-20 }\end{array}$ \\
\hline Yielda (\%) & $70 \pm 12$ & $32.9 \pm 2.7$ & $10.4 \pm 1.0$ \\
\hline Purity (\%) & $29.3 \pm 1.3$ & $42.4 \pm 7.9$ & $93.7 \pm 1.7$ \\
\hline
\end{tabular}

a Yield is calculated by mass compared to the starting mass of the crude green tea extract

$(m / z 169)$, which is in agreement with the HPLC data ( $\bullet$ Fig. $\mathbf{2 d}$ ). In conclusion, we have developed a convenient method for purifying the bioactive flavan-3-ol EGC from a commercial green tea extract. We have used the method to provide sufficient material for bioactivity and mechanistic testing [14]. The method could be scaled up if EGC is found to have bioactivities or properties that warrant production on a larger scale.

\section{Materials and Methods}

$\nabla$

Green tea ( $C$. sinensis) extract was kindly donated by Silvateam. The EGCg standard and tannase powder were provided by Lipton. The EGC standard and Sephadex LH20 were purchased from Sigma-Aldrich. Whatman filter paper (\#40), silica gel (0.060$0.200 \mathrm{~mm}, 60 \AA$ ), and all other chemicals (analytical grade) were purchased from Fisher Scientific. If not otherwise specified, all experiments were performed at room temperature.

Immobilized tannase [15] was prepared by mixing tannase ( $25 \mathrm{~mL}, 0.1 \mathrm{mg}$ protein/mL) with $67 \mathrm{~mL}$ of $3 \%$ aqueous sodium alginate by magnetic stirring for $30 \mathrm{~min}$ and dripping the solution into $1 \mathrm{~L}$ of $2 \% \mathrm{CaCl}_{2}$ with a syringe. Once formed, tannase-con-

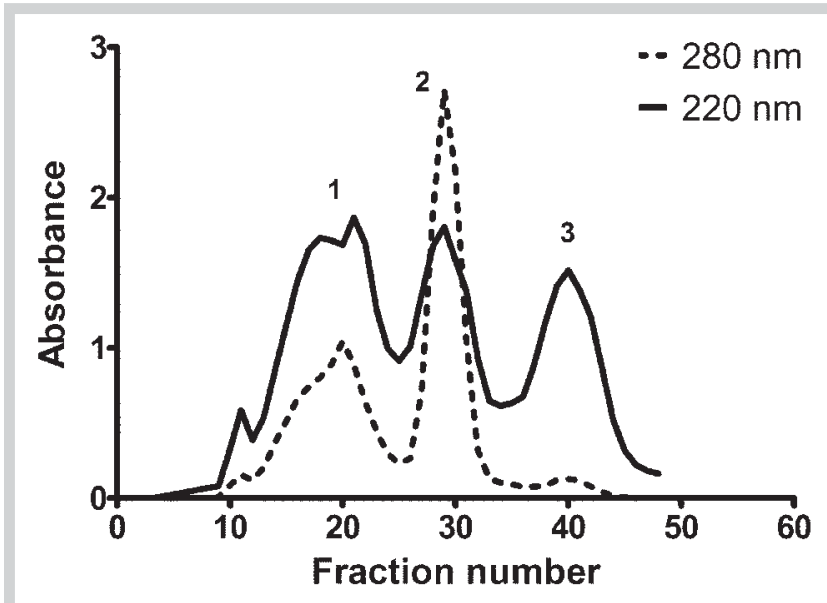

Fig. 3 Isolation and purification of EGC with Sephadex LH-20 chromatography. The absorbance of each sample was measured at both $220 \mathrm{~nm}$ and $280 \mathrm{~nm}$, and the data were plotted against the fraction number. Peak 1 is gallic acid, peak 2 is EGCg, and peak 3 is EGC.

taining beads were transferred to $1 \mathrm{~L}$ of fresh $2 \% \mathrm{CaCl}_{2}$ solution for $1 \mathrm{~h}$ of hardening and were then cross-linked in $1 \mathrm{~L}$ of $0.025 \%$ glutaraldehyde for $2.5 \mathrm{~h}$. After incubation, unreacted glutaraldehyde was removed by rinsing the tannase beads twice with $500 \mathrm{~mL}$ of $50 \mathrm{mM}$ sodium acetate buffer, $\mathrm{pH} 5.0$, before storing the beads in that buffer at $4{ }^{\circ} \mathrm{C}$.

The powdered, dark green crude tea extract $(100 \mathrm{mg}$ ) was mixed with $5 \mathrm{~mL}$ of ethanol with rotation for $10 \mathrm{~min}$ before centrifuging 


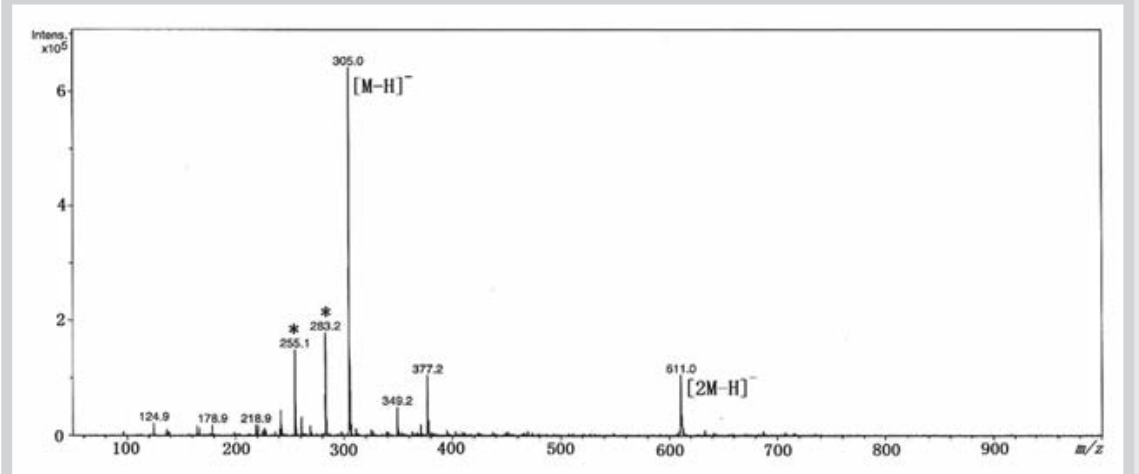

Fig. 4 ESI-MS of purified EGC. *Indicates background signals. MS-MS of the parent ion, 304.9, yielded the following ions (spectrum not shown): $124.9,136.8,138.9,164.8,166.8,178.9,218.9$, 220.9, 261.0. This peak list concurs with that of authentic EGC [13].

for $5 \mathrm{~min}$ at $800 \times \mathrm{g}$. The supernatant was collected and the pellet was resuspended in $5 \mathrm{~mL}$ of ethanol. The extraction was repeated three times in total, with substantial amounts of the original powder dissolving during the extractions. The combined supernatants were rotary evaporated, redissolved in $10 \mathrm{~mL}$ of dichloromethane: ethanol $(50: 50, \mathrm{v} / \mathrm{v})$, and kept at $-20^{\circ} \mathrm{C}$ for purification by silica gel chromatography.

The extract obtained from $100 \mathrm{mg}$ of crude tea was loaded onto a silica gel column $(2 \mathrm{~cm}$ i.d. $\times 25 \mathrm{~cm})$ that was equilibrated with $300 \mathrm{~mL}$ of $85: 15$ dichloromethane: ethanol (v/v). The column was first eluted with $200 \mathrm{~mL}$ of that solvent mixture, and then with $200 \mathrm{~mL}$ of pure ethanol. The last $100 \mathrm{~mL}$ of the ethanol eluate was EGCg-rich and was rotary evaporated to dryness. The residue was redissolved in water, filtered through a double layer of filter paper, lyophilized, and kept at $-20^{\circ} \mathrm{C}$ for tannase-catalyzed hydrolysis.

The lyophilized solid (100 mg) was dissolved in $20 \mathrm{~mL}$ of $50 \mathrm{mM}$ sodium acetate buffer ( $\mathrm{pH} 5.0$ ) and mixed with $\sim 10 \mathrm{~g}$ tannase beads $(\sim 5 \mathrm{~mL})$ plus buffer to achieve a 50 -mL reaction volume. After $30 \mathrm{~min}$ at room temperature, the tannase beads were removed from the solution by filtering the suspension. EGC was enriched by extracting the aqueous filtrate with $100 \mathrm{~mL}$ of ethyl acetate. The ethyl acetate layer was collected, rotary evaporated, reconstituted in water, filtered, lyophilized, and quantified. HPLC was employed to characterize the samples before and after hydrolysis. The lyophilized hydrolysis products were kept at $-20^{\circ} \mathrm{C}$ for isolation and purification with Sephadex LH-20 chromatography.

The lyophilized hydrolysis product $(100 \mathrm{mg})$ was dissolved in $7 \mathrm{~mL}$ of $80 \%$ ethanol containing $0.2 \%$ acetic acid, and was filtered with a $0.22-\mu \mathrm{m}$ nitrocellulose filter. A Sephadex LH-20 column (i.d. $2 \mathrm{~cm} \times 27 \mathrm{~cm}$ ) equilibrated in $80 \%$ ethanol containing $0.2 \%$ acetic acid was used for EGC isolation and purification. A Spectra/Chrom ${ }^{\circledR} \mathrm{CF}-2$ fraction collector was used to collect 50 fractions of eluate ( $8.5 \mathrm{~min} /$ fraction). The absorbance of each fraction was determined at $220 \mathrm{~nm}$ and at $280 \mathrm{~nm}$ with an Agilent 8453 spectrophotometer. Fractions rich in EGC were combined, rotary evaporated, reconstituted in water, lyophilized, and kept at $-20^{\circ} \mathrm{C}$ for HPLC and ESI-MS characterization. The Sephadex LH20 column was cleaned and regenerated with $150 \mathrm{~mL}$ of $80 \%$ ethanol containing $0.2 \%$ acetic acid.

Sample characterization was performed with an Agilent 1100 series HPLC system equipped with a diode array detector (Agilent Technologies) and Chemstation A.09.03 software. Separation was performed on a Zorbax Eclipse XD8 C8 column $(5 \mu \mathrm{m}$, $4.6 \times 150 \mathrm{~mm}$ i.d.) at a flow rate of $0.5 \mathrm{~mL} / \mathrm{min}$. Samples were eluted with a gradient of $0.1 \%$ trifluoroacetic acid in water (sol- vent $\mathrm{A}$ ) and $0.13 \%$ trifluoroacetic acid in acetonitrile (solvent $\mathrm{B}$ ) as follows: $0-10 \mathrm{~min}, 5 \% \mathrm{~B} ; 10-20 \mathrm{~min}, 20 \% \mathrm{~B} ; 20-30 \mathrm{~min}, 35 \%$ B; 30-33 min, 95\% B; 33-35 min, 5\% B. Samples were filtered through $0.22 \mu \mathrm{m}$ cellulose acetate spin filters before injecting $10 \mu \mathrm{L}$ on the HPLC. The product EGC $\left(t_{\mathrm{R}} 19.6 \mathrm{~min}\right)$ and starting material EGCg $\left(t_{\mathrm{R}} 24.7 \mathrm{~min}\right)$ were detected using the diode array detector to collect data from $200 \mathrm{~nm}$ to $400 \mathrm{~nm}$. The spectrum of each peak was generated with the Chemstation software.

Mass spectra were obtained with an Esquire LC ion trap instrument (Bruker Daltonics) with Data Analysis 3.0 software. The ESI source was operated in the negative mode. A nominal target mass was set to 306 before fine-tuning. The capillary, skimmer 1, and trap drive voltages were $100.2,-29.2$, and $46.1 \mathrm{~V}$, respectively. The ion charge control was on with a target of 20000 . The $300^{\circ} \mathrm{C}$ nitrogen dry gas flow rate was $4 \mathrm{~L} / \mathrm{min}$ and the nebulization gas pressure was $11 \mathrm{psi}$. A syringe pump was used to infuse the sample at $10 \mu \mathrm{L} / \mathrm{min}$. Each data point in the spectrum consisted of an average of 20 scans over a mass range of 50 to $1000 \mathrm{~m} / \mathrm{z}$.

The yield (mass) and purity (HPLC) of the product were calculated from four independent replications of the purification, and are reported as the average $\pm \mathrm{SD}$. The purity was determined by comparing the integrated peak areas of EGC or EGCg to calibration curves prepared with the respective authentic standard.

\section{Acknowledgements}

$\nabla$

This work was partially funded by NIDDK R15DK069285.

\section{Conflict of Interest}

$\nabla$

The authors declare no conflict of interest.

\section{References}

1 Nakagawa T, Yokozawa T, Terasawa K, Shu S, Juneja LR. Protective activity of green tea against free radical- and glucose-mediated protein damage. J Agric Food Chem 2002; 50: 2418-2422

2 Wheeler DS, Wheeler WJ. The medicinal chemistry of tea. Drug Dev Res 2004; 61: 45-65

3 Chen S, Osaki N, Shimotoyodome A. Green tea catechins enhance norepinephrine-induced lipolysis via a protein kinase A-dependent pathway in adipocytes. Biochem Biophys Res Commun 2015; 461: 1-7

4 Xia GB, Hong S, Liu SB. Simultaneous preparation of naturally abundant and rare catechins by tannase-mediated biotransformation combining high speed counter current chromatography. Food Chem 2014; 151 : 380-384

5 Vuong QV, Stathopoulos CE, Nguyen MH, Golding JB, Roach PD. Isolation of green tea catechins and their utilization in the food industry. Food Rev Int 2011; 27: 227-247 
6 Bazinet L, Labbe D, Tremblay A. Production of green tea EGC- and EGCgenriched fractions by a two-step extraction procedure. Sep Purif Technol 2007; 56: 53-56

7 Amarowicz R, Shahidi F, Wiczkowski W. Separation of individual catechins from green tea using silica gel column chromatography and HPLC. J Food Lipids 2003; 10: 165-177

8 Amarowicz R, Shahidi F. A rapid chromatographic method for separation of individual catechins from green tea. Food Res Int 1996; 29: 71-76

9 Cao XL, Ito Y. Preparation and purification of epigallocatechin by highspeed countercurrent chromatography (HSCCC). J Liq Chromatogr Relat Technol 2004; 27: 145-152

10 Rehm BHA. Alginates: Biology and Applications. Berlin: Springer; 2009: 266

11 Daneshfar A, Ghaziaskar HS, Homayoun N. Solubility of gallic acid in methanol, ethanol, water, and ethyl acetate. J Chem Eng Data 2008; 53: 776-778

12 Li N, Taylor LS, Ferruzzi MG, Mauer LJ. Kinetic study of catechin stability: effects of pH, concentration, and temperature. J Agric Food Chem 2012; 60: 12531-12539

13 Global Natural Products Social Molecular Networking (GNPS). GNPS Library Spectrum CCMSLIB00000081482 (Negative ion mode ESI MS/MS EGC). Available at https://gnps.ucsd.edu/ProteoSAFe/ gnpslibraryspectrum.jsp?SpectrumID=CCMSLIB00000081482\&\#\%7B\% 7D. Accessed April 6, 2016

$14 \mathrm{Li} \mathrm{M}$, Hagerman AE. Role of the flavan-3-ol and galloyl moieties in the interaction of (-)-epigallocatechin gallate with serum albumin. J Agric Food Chem 2014; 62: 3768-3775
15 Su E, Xia T, Gao L, Dai Q Zhang Z. Immobilization and characterization of tannase and its haze-removing. Food Sci Technol Int 2010; 15: 545552

received February 15, 2016

revised April 21, 2016

accepted May 21, 2016

Bibliography

DOI http://dx.doi.org/10.1055/s-0042-109780

Planta Med Int Open 2016; 3: e35-e38

(c) Georg Thieme Verlag KG Stuttgart · New York .

ISSN 2509-6656

\section{Correspondence}

\section{Prof. Ann E. Hagerman}

Department of Chemistry \& Biochemistry

Miami University

651 E. High Street

Oxford, OH 45056

United States

Phone: + 15135292827

Fax: + 15135295715

hagermae@miamioh.edu

License terms

()ㅜ (1) $\ominus \circledast$ 